x 
Bewohnte Natur Living with Nature 
Sandra Hofmeister

Hinaus aufs Land!

Warum es Städter in die Natur zieht

Let's go to the countryside!

Why city dwellers are drawn to nature 007

\section{Jakob Schoof}

Von der Subsistenzwirtschaft

zur Sommerresidenz

Eine kurze Geschichte des Wohnens

auf dem Land

From subsistence economy

to summer residence

A short history of living

in the country

133
Wespi de Meuron Romeo

Betonhaus, Brissago (CH)

Concrete House, Brissago $(\mathrm{CH})$

024

\section{ACME}

Hunsett Mill, Stalham (GB)

Hunsett Mill, Stalham (GB)

030

ColoradoBuildingWorkshop

Mikrohütten, Leadville, Colorado (US)

Micro Cabins, Leadville, Colorado (US) 036

christian pottgiesser architecturespossibles Maison L, île-de-France (FR)

Maison L, Île-de-France (FR)

042

Bernardo Bader Architekten

Haus am Moor, Krumbach (AT)

House on the Moor, Krumbach (AT)

046

Crosson Architects

Hütte auf Kufen, Whangapoua (NZ)

Hut on Sleds, Whangapoua (NZ)

052

Tham \& Videgård Arkitekter

Sommerhaus, Lagnö (SE)

Summer House, Lagnö (SE)

058

Witherford Watson Mann Architects

Astley Castle, Nuneaton (GB)

Astley Castle, Nuneaton (GB)

064

Tegnestuen Vandkunsten

Tanghus, Laesø (DK)

Tanghus, Laesø (DK)

068

Cristián Izquierdo

Casa en Morillos, Coquimbo (CL)

Casa en Morillos, Coquimbo (CL)

072 
Morger Partner Architekten

Stallhaus, Lumbrein $(\mathrm{CH})$

Stable House, Lumbrein $(\mathrm{CH})$

078

Leth \& Gori

Dachhaus, Fredensborg (DK)

Roof House, Fredensborg (DK)

082

dekleva gregorič architects

Karsthaus, Vrhovlje (SI)

Karst House, Vrhovlje (SI)

086

Arquitectura-G

Casa Luz, Cilleros (ES)

Casa Luz, Cilleros (ES)

090

Aslak Haanshuus Arkitekter

Hytte Femunden, Røros (NO)

Hytte Femunden, Røros (NO)

096

SAMI-arquitectos

E/C House, São Miguel Arcanjo, Insel Pico (PT)

E/C House, São Miguel Arcanjo, Pico Island (PT) 102

Ofis arhitekti

Winterhütte, Bovec (SI)

Winter Cabin, Bovec (SI)

108

PK Arkitektar

Ferienhäuser, Brekkuskógur (IS)

Holiday Cottages, Brekkuskógur (IS)

114

\section{Studio mk27}

Casa na Mata, Guarujá, São Paulo (BR)

Casa na Mata, Guarujá, São Paulo (BR)

120

Jarmund/Vigsnæs Arkitekter MNAL

Rabot Touristenhütte, Okstindan Range (NO)

Rabot Tourist Cabin, Okstindan Range (NO)
Autoren

Authors

150

Adressen

Addresses

151

Bildnachweise

Picture credits

152

Impressum

Imprint

153 
